\title{
Reflections on Methods from an Interdisciplinary Research Project in Global Environmental $\mathrm{Law}^{\Psi}$
}

\author{
Louisa Parks* and Elisa Morgera**
}

\begin{abstract}
This research note reflects on the methods (as distinct from methodology) used in a five-year interdisciplinary and multi-site research project in global environmental law, and their links to questions of research ethics. We highlight the iterative processes that proved necessary to compare five case studies on local communities engaged in varied discussions on fair and equitable benefit sharing in different regions of the world and their implications for international environmental law. The note recommends explicit reflection on research methods and ethics to acknowledge and address power relationships in global environmental law research.
\end{abstract}

Keywords: International environmental law, global environmental law, research methods, research ethics, comparative case studies.

\section{INTRODUCTION}

This research note reflects on the challenges experienced and lessons learnt during a five-year, interdisciplinary and multi-site research project to investigate fair and equitable benefit sharing from a global environmental law perspective. ${ }^{1}$ We understand global environmental law as a sub-set of transnational environmental law, namely, as legal phenomena that not only transcend national frontiers but also have a global justification. ${ }^{2}$ This is the case, for example, of an international environmental treaty objective (fair and equitable benefit-sharing) the realization of which relies on a variety of different legal orders. ${ }^{3}$ Benefit sharing, while enshrined in a variety of international

\footnotetext{
${ }^{\Psi}$ This contribution is part of a collection of articles growing out of the conference 'Global Environmental Law’, held at Strathclyde Law School, Glasgow (United Kingdom), on 4-5 Sept. 2017.

* University of Trento, School of International Studies and Department of Sociology and Social Research, Trento (Italy).

Email: louisa.parks@unitn.it.

${ }^{* *}$ University of Strathclyde, Glasgow (United Kingdom).

Email: elisa.morgera@strath.ac.uk.

${ }^{1}$ BeneLex, Benefit-sharing for an equitable transition to the green economy - the role of law, funded by the European Research Council (grant 335592).

${ }^{2}$ Drawing on N. Walker, Intimations of Global Law (Cambridge University Press, 2015), pp.15-8.

${ }^{3}$ E. Morgera, 'Bilateralism at the Service of Community Interests? Non-judicial Enforcement of Global Public Goods in the Context of Global Environmental Law' (2012) 23 European Journal of International Law, pp. 743-767.
} 
treaties, ${ }^{4}$ in effect relies for its further development and implementation on a dynamic web of national laws, contractual arrangements and communities' customary laws. ${ }^{5} \mathrm{~A}$ global environmental law perspective thus prompts the study of environmental law at the international, regional, national and sub-national levels as a set of inter-related and mutually influencing systems, through an analysis of the cumulative impacts of the practice of a variety of non-state actors. ${ }^{6}$ It seeks to 'gauge incipient trends and identify future projections, in an iterative process of mapping, scanning, schematizing and (re)framing normative patterns, with a view to understanding the capacity of law ... to recast the ways in which it addresses some of the problems of an interconnected world'. ${ }^{7}$

Our focus here is on research methods, as our experience revealed the intricate links between methods for local community-based research and questions of research ethics which have not been discussed in the incipient literature on global environmental law. We distinguish methods from methodology ${ }^{8}$--- which is also occasionally lacking in the debate on environmental legal scholarship ${ }^{9}-$ in line with the literature on political and social research design. We understand methodology as a 'toolbox' that can be filled with various tools, which are the research methods. ${ }^{10}$ Methodology flows from ontological, epistemological and theoretical framework decisions that derive from the research interests of the researcher. In that sense, methodological discussions answer questions about the best overall way of acquiring knowledge about a research question. In this view, methods are not the automatic outcomes of any one particular methodology; a constructivist piece of research may draw on quantitative methods, just as a positivist piece of research may use qualitative ones. ${ }^{11}$ The mixture of traditional

\footnotetext{
${ }^{4}$ See generally E. Morgera, M. Buck, \& E. Tsioumani (eds), The Nagoya Protocol in Perspective: Implications for International Law and Implementation Challenges (Martinus Nijhoff, 2012).

${ }_{6}^{5}$ Morgera, n. 3 above, p. 761.

${ }^{6}$ Ibid, p. 746.

${ }^{7}$ Walker, n. 2 above, pp. 126, 148-77.

${ }^{8}$ See Section 2. We address methodology in more depth elsewhere. See E. Morgera, L. Parks \& M. Schroder, 'Methodological Challenges of Transnational Environmental Law' in V. Heyvaert \& L-A. Duvic-Paoli (eds), Research Handbook of Transnational Environmental Law (Edward Elgar, Forthcoming 2019).

${ }^{9}$ E. Fisher, B. Lange, E. Scotford \& C. Carlane, 'Maturity and Methodology: Starting a Debate about Environmental Law Scholarship' (2009) 21 Journal of International Law, pp. 213-250; and generally A. Philippopoulous-Mihalopoulos \& V. Brooks (eds), Research Methods in Environmental Law: A Handbook (Edward Elgar, 2017).

${ }^{10} \mathrm{~J}$. W. Moses \& T. L. Knutsen, Ways of Knowing: Competing Methodologies in Social and Political Research (Palgrave Macmillan, $2^{\text {nd }}$ ed, 2012).

${ }^{11}$ Ibid.
} 
legal methods and of quantitative and qualitative social scientific methods used in our project illustrates this. ${ }^{12}$ Methodology (and preceding questions of ontology and epistemology) also raises ethical considerations. Because this is already covered in a great deal of literature at the stage of research design, ${ }^{13}$ we focus on the less discussed area of ethical decision making as research unfolds.

We thus first provide an overview of the research design process and describe the moves from the initial ontological standpoint through to methodology. This serves to explain the choice of common social science methods we used to study benefit sharing at different levels and in different sites (Section 2). We then discuss the importance of an explicit discussion of, and of an iterative approach to, methods in the study of global environmental law (Section 3). On that basis, we link the choice of research methods to ethical questions (Section 4). We finally discuss how our iterative view of methods and ethics fed back into methodological considerations and produced new research questions (Section 5).

\section{RESEARCH DESIGN: FROM METHODOLOGY TO METHODS}

The ontological starting point for our project is constructivist: we see meaning as socially constructed in interactions between different actors. This applies to the arenas where international environmental law is made and successively interpreted by state and non-state actors. Such actors include representatives of local communities operating at the global level, and the communities and other actors that apply international environmental legal norms in a local context. We believe this is a reasonable starting point for fair and equitable benefit sharing for two reasons. First, benefit sharing is a legal concept that is still subject to progressive development at both international and local levels. Second, benefit sharing, as progressively articulated in international environmental law, arguably supports equitable partnership building

\footnotetext{
${ }^{12}$ L. Parks \& E. Morgera, 'The Need for an Interdisciplinary Approach to Norm Diffusion: The Case of Fair and Equitable Benefit-sharing' (2015) 24 Review of European Community \& International Environmental Law, pp. 353-367.

${ }^{13}$ See, e.g., P, Reason \& H. Bradbury, The Sage Handbook of Action Research (Sage, 2008); Moses and Knutsen, n. 10 above.
} 
among state and non-state actors across different sectors (bio-prospecting, conservation, natural resource use and the production of knowledge). ${ }^{14}$

As our interest was in a specific concept of international environmental law and its development at multiple levels, a constructivist view allowed us to pay attention to the negotiation of meaning. This led us in turn to an interpretivist epistemology. In this vein, we understand relevant knowledge about fair and equitable benefit sharing to exist not only in sources of law, but also in real-life applications and actors' viewpoints.

To respond to these ontological and epistemological premises, we drew on theories of norm diffusion from law, international relations and political sociology. We considered how each of these approaches contributes to illuminate different ways of constructing the meaning of benefit sharing in different sites - that is how the law lives and evolves over time by travelling across sites. ${ }^{15}$ The existing literature from these three social scientific disciplines underlines that politics and law play crucial roles in how norms diffuse. It equally shows how norms are framed or adjusted to different social and cultural contexts.

This interdisciplinary theoretical approach underpinned our choice of a comparative case-study methodology to consider both legal sources and the social and political worlds associated with fair and equitable benefit sharing, and its application or discussion at different levels. This choice was also motivated by the fact that the bulk of the literature on benefit sharing focuses either on single local-level cases or sectors, or on negotiation and application via international instruments. Starting our exploration of the meanings constructed around benefit sharing at the local level, we chose a 'most different systems' comparative case-study methodology so that any common traits arising from the comparison would be more robust, albeit not generalizable. We decided that a total of five case studies was feasible to allow enough space for variation and comparison yet still yield in-depth knowledge. ${ }^{16}$

\footnotetext{
${ }^{14}$ E. Morgera, 'The Need for an International Legal Concept of Fair and Equitable Benefit-sharing' (2016) 27 European Journal of International Law, pp. 353-383. See also Parks \& Morgera, n. 13 above. ${ }^{15}$ Parks \& Morgera, n. 13 above.

${ }^{16}$ D. Della Porta, 'Comparative Analysis: Case-oriented versus Variable-oriented Research' in D. Della Porta \& M. Keating (eds), Approaches and Methodologies in the Social Sciences: A Pluralist Perspective (Cambridge University Press, 2008), pp. 198-222.
} 
The choice of the individual case studies was driven by our constructivist viewpoint: to unravel how benefit sharing was framed. That is, we sought to uncover how its meaning was constructed in different regions and domestic legal systems (Greece, South Africa, Namibia, Malaysia, and Argentina), in different sectors which raise different environmental concerns (pastoralism, traditional medicine, protected areas, traditional agriculture, and mining), and within communities with different degrees of access to power at different levels. ${ }^{17}$

We selected local communities that were discussing community protocols (although not all had drafted or adopted then), taking this to signal the presence of discussions about benefit sharing in a broad sense. Community protocols are 'documents developed by local communities to record, among other things, information on their practices, institutions, customary law, rights and visions of development'. ${ }^{18}$ They are drafted, usually with external support, to set out a community's position on how it expects any external actor to seek to access resources to traditional lands, resources and knowledge. Discussions around community protocols are likely to highlight what a community might expect or demand in building a relationship with outside actors, as a starting point for any potential future discussions on benefit sharing.

\subsection{Trade-offs in Research Design Choices}

A comparative case study methodology involves some common trade-offs linked to the choice between depth and breadth. This underpinned the decision to work with nongovernmental organizations (NGOs) that were, in turn, working closely with local communities. Ideally, we would have developed our own relationships with communities over time in an ethnographic, participatory approach. However, in order to cover five cases in five years, we relied on NGOs to help us identify communities discussing issues around benefit sharing (guaranteeing the variation discussed earlier). The NGOs also helped us learn about those communities and take informed decisions

\footnotetext{
${ }^{17}$ The case studies are described in more depth in L. Parks 'Challenging power from the bottom up? Community protocols, benefit sharing, and the challenge of dominant discourses' (2018) 88 Geoforum $87-95$, at $87-88$.

${ }^{18}$ Ibid; see also Parks \& Morgera, n. 13 above.
} 
on how to approach them respectfully prior to our research visits. They also assisted in selecting the most appropriate research methods and consent processes.

The decision to work with NGOs also produced knock-on effects: working with other actors to identify and carry out research meant that our views on communities were necessarily shaped by, and filtered through, the relationship between community and NGO. On the one hand, a substantial body of literature on civil society notes that NGOs may become (albeit unwittingly) actors in the expansion of neoliberal systems seen as damaging to many communities, for example by allowing states to avoid providing vital services, or by creating dependencies between communities and NGOs. ${ }^{19}$ On the other hand, not enough research has focused on the role of NGOs as facilitators of negotiations on community protocols and their role in influencing the meaning of benefit sharing in local contexts through that support. We thus sought out NGOs involved closely with communities in long-term projects and relationships aimed at local empowerment rather than service provision. These NGOs were aware of the potential for civil society groups to create dependencies and better placed to advise us about how to feed into ongoing community support (as discussed in more depth later). ${ }^{20}$ The NGO partners made preliminary investigations on our behalf to determine whether communities would be potentially interested in participating in the research. We also decided to include two visits to each local community in order to build in a feedback loop, which would allow us to reflect on the evolving relationship between the NGO and community over time.

\section{REFLECTIONS ON METHODS FOR STUDYING GLOBAL ENVIRONMENTAL LAW}

Our interdisciplinary theoretical framework informed the methods - that is, the tools for carrying out a comparative case-study methodology - which we employed to study global environmental law within local-community discussions around benefit sharing.

\footnotetext{
${ }^{19}$ M. Kaldor, Global Civil Society: An Answer to War (Polity Press, 2003); A. A. Choudry \& D. Kapoor, NGOization: Complicity, Contradictions and Prospects (Zed Books, 2013).

${ }^{20}$ E. Pittaway, L. Bartolomei \& R. Hugman, 'Stop Stealing Our Stories: The Ethics of Research with Vulnerable Groups’ (2010) 2 Journal of Human Rights Practice, pp. 229-251.
} 
Our methods, in turn, shaped research in the project within different disciplines. ${ }^{21}$ They enriched more traditional (doctrinal) legal research, and gave rise to a politicalsociological analysis of international environmental law. ${ }^{22}$

Despite the common use of these methods across social science disciplines, we have seen relatively little literature in the legal field discussing ontological, epistemological and methodological considerations around the choice of research methods. ${ }^{23}$ More generally, we were also unable to locate literature on practical and ethical aspects of the methods choices when applied in empirical, exploratory research on international law at the local level. Practical issues are discussed in this section, with a view to underscoring the importance of methods choices to limit bias in global environmental research, ${ }^{24}$ and the need for an iterative approach.

Doing empirical research is always a balancing act to some extent. Researchers must juggle practical concerns, faithfulness to research design, and the on the-spot-decisionmaking that empirical research may demand. These common issues were magnified in our comparative case-study methodology as a result of the most-different-systems design. This meant that each case study presented a range of context-specific opportunities and challenges. We anticipated practical difficulties and sought to remain flexible regarding which methods we would use in our comparative case study methodology at the research design stage. ${ }^{25}$ This allowed us to link our choice of methods with discussions with partner NGOs.

\footnotetext{
${ }^{21}$ For an in-depth discussion of inter- and multi-disciplinarity within the research project, see Morgera, Parks \& Schroder, n. 8 above.

${ }^{22}$ L. Parks, 'Spaces for local voices? A discourse analysis of the decisions of the Convention on Biological Diversity' (2018) 9 Journal of Human Rights and the Environment, pp. 141-170.

${ }^{23}$ But see G. A. Sarfaty, 'International Norm Diffusion in the Pimicikamak Cree Nation: A Model of Legal Mediation’ (2007) 48 Harvard International Law Journal, pp. 441-482; E. Lees \& J. E. Vinuales, The Oxford Handbook of Comparative Environmental Law (Oxford University Press, 2019); Philippopoulos-Mihalopoulos \& Brooks, n. 9 above.

${ }^{24}$ This was considered a serious flaw in global law research more generally by W. Twining, 'Diffusion of Law: A Global Perspective' (2004) 36 Journal of Legal Pluralism and Unofficial Law 1-45; for discussion in an environmental law context, see E. Morgera, 'Global Environmental Law and the Comparative Legal Method(s)' (2015) 24 Review of European Community and International Environmental Law (RECIEL), pp. 254-263, at 258 and 263.

${ }^{25}$ L. Parks and E. Morgera, 'An Inter-disciplinary Methodology for Researching Benefit-sharing as a Norm Diffusing in Global Environmental Law' (SSRN, 2014) BENELEX Working Paper No 2. Available at https://papers.ssrn.com/sol3/papers.cfm?abstract id=2524333.
} 
We considered a range of qualitative methods feasible for gathering data about the meanings attributed to benefit sharing: focus groups, participant observation, and semior unstructured interviews. ${ }^{26} \mathrm{We}$ reasoned that qualitative methods were the best suited to our research interests, since they are used 'to make sense of, or to interpret, phenomena in terms of the meanings people bring to them'. ${ }^{27}$ The option of focus groups was discarded after discussions with NGO partners. Despite the many advantages of this method for understanding meaning construction in a dynamic setting, focus groups were simply too complex to set up with often remote communities. They require long run-up periods to organize and explain the purpose of the groups. This highlights a specific trade-off in terms of the methods flowing from our overall research design. Instead, we relied on other common methods - participant observation at community meetings, workshops and festivals, and individual and group interviews (informal conversations or semi-structured interviews).

Participant observation was our preferred method for gathering information about how communities discuss and assign meaning to benefit sharing. This was for several reasons, both practical and epistemological. In terms of the kind of knowledge we wished to gather, participant observation allowed us to observe interactions and conversations over longer timeframes than was possible with interviews. In addition, they provided an opportunity to see exchanges between community members that bore a closer resemblance to natural and spontaneous exchange. ${ }^{28}$ Although the researcher's presence inevitably has some distorting effect, these are arguably less prominent than in interview scenarios. ${ }^{29}$ In our research in particular, this lower level of researcher distortion was supported by our choice to play a more passive role, without imposing the term 'benefit sharing' or our own understanding of it (mainly based on previous study of international environmental law) on community discussions. Our presentations of the research project and benefit sharing were more limited where we used the participant observation method: we relied on the initial contacts made through the

\footnotetext{
26 Ibid.

${ }^{27}$ D. Della Porta, 'How Many Approaches in the Social Sciences? An Epistemological Introduction', in D. Della Porta \& M. Keating (eds), Approaches and Methodologies in the Social Sciences: A Pluralist Perspective (Cambridge University Press, 2008), pp. 19-39.

${ }^{28}$ See, e.g., U. Flick, An Introduction to Qualitative Research (Sage, 2014), p. 317.

29 See, e.g., K. M. Blee \& V. Taylor 'Semi-Structured Interviewing in Social Movement Research', in B. Klandermans \& S. Staggenborg (eds), Methods of Social Movement Research (University of Minnesota Press, 2002), pp. 92-117.
} 
partner NGO and briefly presented our research interests orally before requesting communities' permission to observe meetings. We also relied on our partner NGOs to schedule our research visits to coincide with community events where discussions around benefit sharing were likely to take place.

Overall, we considered that participant observation at meetings or gatherings where community members had already decided to discuss issues linked to benefit sharing would allow us to gain an understanding of how meanings were assigned to this idea, as well as the kinds of issues that were raised around it. This we deemed crucial to understanding local framings of benefit sharing in contrast to those emerging from international environmental law processes. This stance tied into our comparative casestudy methodology rooted in turn in a constructivist view of law and social interaction.

Participant observation was not always possible, since not all communities involved in our research project were organized in formal ways or held community meetings. Where these conditions were not in place, we held informal, unstructured interviews with community members, and more formal, semi-structured interviews with community members holding official positions.

Our choice of less structured interviews with community members was guided by our overall constructivist research design. We dispensed with any checklist of areas to be covered, since we wanted to allow views of benefit sharing to emerge in interaction with as little pre-determined shaping of the discussion as possible. The latter is also a general point of method. As discussed, distortion of the discussion was something we wished to reduce wherever possible, to limit our bias (based on previous research in international environmental law) of local-level perceptions and framings of benefit sharing. By holding informal interviews more akin to everyday conversations, we hoped to minimize any boundaries placed on the conversation and allow interview partners to tell their own stories. This form of interview minimizes the "voice of the researcher' as much as possible, and brings to the fore the aspects of human agency central to our understanding of the law's meaning as built in social interaction. ${ }^{30}$ From a practical perspective, informal and unstructured interviews also allowed us to be more

\footnotetext{
${ }^{30}$ Ibid.
} 
spontaneous, speaking with local community members as opportunities arose. This was simply common sense in areas where research participants were not easily contactable and whose work meant availability was unpredictable.

Unstructured interviews were less practical for participants working for different authorities or in other organizations with supporting roles in communities or alternative connections of interest. Here, we used semi-structured interviews, since most of these participants had prior knowledge of benefit sharing in relation to their official mandate. This allowed us more leeway in terms of balancing the distortion of the conversation and setting boundaries in line with the research questions. ${ }^{31}$

On some occasions we were unable to use any of these methods - yet these also informed our research. For example, in some instances we were asked not to observe meetings or were denied interviews. These denials were informative about sensitivities and risks around benefit sharing in some of the cases, and ultimately helped us gain a deeper understanding of the multiple interests of different actors in communities that benefit-sharing is expected to address. In Namibia, for example, we observed the presentation of a draft community protocol in various villages. As this was only a draft, however, we withdrew as communities discussed their opinions on the details of the text. This, along with other experiences, drew attention to the importance of community 'ownership' over community protocols, and the processes of consensus-building involved in their drafting. On many more occasions, meetings simply fell through. This provided us with a first-hand understanding of the challenges faced in many of the communities we visited, ranging from a lack of access to transport, to a lack of communication possibilities and the unpredictable nature of work. These barriers are highly relevant to our research interests since access to information, and the possibility to organize as a community, are central factors for the implementation of fair and equitable benefit sharing. Our expectations about cultural and language barriers were also challenged: these were steeper than we imagined in our only European case study in Greece for example, where few interview partners spoke English, and where

\footnotetext{
${ }^{31}$ See, e.g., C. Frankfort-Nachmias \& D. Nachmias, Research Methods in the Social Sciences (St. Martin's Press, 1992).
} 
pastoralists worked long hours making late night interviews necessary. In other case studies, these barriers were less pronounced than we had anticipated.

These reflections serve to highlight that as we carried out our research, unexpected elements resulted in tweaks, adjustments and occasional changes in the methods we had intended to use. Yet we decided that adapting our methods to be as undisruptive, respectful and ultimately useful to research partners as possible was more important than forcing a neat and uniform approach to facilitate our scholarly comparison. What this means is that the research process in global environmental law is an iterative one, negotiated with research partners in the moment, rather than neatly laid out in prior plans. $^{32}$ Flexibility in the choice of methods, as well as explicit reflection on their respective merits and drawbacks, emerges as an essential element for studying the surprising and unpredictable meanings of benefit sharing constructed at different levels (with various constraints and idiosyncrasies), and by various actors (with different cultural and political sensitivities).

\section{ETHICS}

Questions of ethics, which we consider as comprising questions of power and consent, should be addressed when specifying the ontological, epistemological and methodological aspects of a research design. In our experience, however, attention should also be paid to questions of ethics that arise while applying social science methods 'on the ground'. These are questions that emerge after designing the research and after fulfilling the necessary research ethics procedures applicable in a particular case.

In this section we cover two sets of ethics questions in particular. Firstly, we reflect on power relationships in questions of method and ethics in local-level research. In particular, we discuss how we drew on participatory action research (PAR) and its emphasis on power and self-reflexive research approaches, which opened our eyes to the complex webs of power in which communities are embedded, including

\footnotetext{
${ }^{32}$ S. Vermeylen \& G. Clark, 'An Alternative Ethics for Research: Levinas and the Unheard Voices and Unseen Faces’ (2017) 20 International Journal of Social Research Methodology, pp. 499-512.
} 
relationships with supporting NGOs and researchers. Secondly, we reflect on the gap between formal ethics procedures and ethical research on the ground, illustrating the shortcomings of written consent requirements and the need for dialogue-based ethics processes. In both areas, we build upon our earlier reflections on the need to address researchers' bias and adopt iterative approaches in the choice of research methods.

\subsection{Power}

Although PAR inspired our approach to ethics, we do not claim to have carried out PAR (which involves the co-development of research designs alongside those usually characterized as the 'researched'). Whether the funding modalities for our project would have allowed such a co-design process in the first place remains an open question. Instead, PAR provided us with a template to consider power relationships in our own research, and to move towards integrating the views of research partners into the aims ${ }^{33}$ and modalities of the project iteratively as it progressed. Our research became more self-reflexive as it unfolded, drawing on our substantive findings about the importance of the definition of benefits in dialogue with communities, as well as on our iterative approach to methods and the need to address researchers' bias. This also ties in with our earlier reflections on the role of partner NGOs in our research design.

In practice, we followed PAR insights at the research planning stage in an effort to build our sensitivity to power imbalances between researchers and participants in dialogue with partner NGOs. During our research, however, the variety of constellations of power we needed to consider proved more complex and wide-ranging than we had imagined. We had thought through strategies that might diminish power imbalances between researchers and participants, and opted for less disruptive methods. Doing the research made it clear that these relations were embedded in local, regional, national, and international power relationships in clear everyday ways.

In South Africa, for example, we found that traditional health practitioners were dealing with traditional and municipal authorities, as well as the ministry of health, in an effort to gain recognition. They were also engaged in talks with provincial parks authorities

\footnotetext{
${ }^{33}$ Reason \& Bradbury, n. 13 above.
} 
and private landowners around access issues, complicated in turn by ongoing land claims and serious security problems linked to global rhinoceros poaching. Added to this, the national legislative backdrop was shifting with the implementation of international law. In this context, while the researcher-participant power relationship remained important, it was only one among many. Our research approaches and, in particular, our ethics commitments thus needed to be responsive to a range of different contexts within each case study, and were refined in an iterative manner. Our methods choices were thus tailored not only to the levels of organization in local communities, but to sensitivities linked to power constellations. For example, we did not observe entire community meetings in some cases, and held group interviews in others, in an effort to ensure that no community member felt their views were excluded.

This brings our discussion to another important power relationship - that with NGO partners. The decision to work with partner NGOs creates the possibility that working with and through these actors influences research findings, since NGOs are also part of complex contexts of power around communities. NGOs have been thought of as 'gatekeepers' in social scientific research, and thus as a potential source of distortion in research. We addressed this in terms of ethics in two ways, one more and one less intentional. Firstly, we consciously reflected on the role of NGOs in our research, and took steps to make this challenge explicit in our findings. ${ }^{34}$ Secondly, and less intentionally, our decision to use discussions of community protocols as an indicator for a community's interest in benefit sharing helped to mitigate the shaping of our results through community-NGO relationships. Open and participatory practices are crucial to the elaboration of community protocols, and the effort and thought our NGO partners put into facilitating community-owned processes was remarkable. Though this is a difficult balancing act, ${ }^{35}$ it meant that the NGO partners we worked with were aware of their power, reflected upon it, and sought measures to address inequalities, not least through their aim of community empowerment. To do ethical research with these NGOs also meant seeking to protect these relationships aimed at empowerment. We avoided some themes or approaches that NGOs deemed risky to those relationships, and

\footnotetext{
${ }^{34}$ E.g Parks, n. 22 above.

35 Ibid.
} 
contacted some representatives of authorities independently from NGOs for the same reason.

The intractable problem of power can never be entirely resolved, but some steps can be taken. They include working with actors that dedicate time and thought to these issues and being explicit about their existence and possible effects on research results. This can go some way to mitigate, or at least make explicit, some aspects of an unequal relationship, and to contribute to conversations about appropriate new research directions and methods. Recent literature highlights that power must be tackled in reflexive ways in each research encounter. ${ }^{36}$ This view is echoed in literature that discusses 'gatekeepers' as more than actors who simply facilitate access, but instead shape and contribute to relationships of power and more, and who are integral to the whole research process. ${ }^{37}$ Thinking of research partners in this way shifts this power relationship into the research ethics domain, and raises the idea that ethical research should also be responsive (where possible) to the needs of research participants. When reflecting on how to shape our research in ways that would respond to communities' interests, we asked NGO partners - which we considered as part of the power context around communities - if and how we could contribute to their work of supporting and empowering communities, including those beyond the scope of our research. While we were not always able to satisfy all requests, for example on specific points of national law which were beyond our expertise, we were able to find some ways forward in dialogue. For instance, we identified, in response to community concerns, how international law could be used in the local context to challenge national approaches that did not respond to community views and needs that had been internationally recognized.

Our complex relationships with NGOs and communities also helped us reflect on the communication of research findings in ways that would, hopefully, be immediately actionable by research partners, even where they addressed their concerns only in part. In some cases, research partners remained skeptical that international law and relevant research findings would be helpful to them. This too was an important finding which,

\footnotetext{
${ }^{36}$ Vermeylen \& Clark, n. 32 above.

${ }^{37}$ I. Crowhurst \& M. Kennedy-Macfoy, 'Editorial. Troubling Gatekeepers: Methodological Considerations for Social Research' (2013) [16] International Journal of Social Research, pp. 457-462.
} 
without this kind of cooperation, might not have been discovered and contributed to address researchers' biases. ${ }^{38}$ In one of our case studies, communities did not wish to discuss benefit sharing as such, as their own conversations focused on environmental impact assessments and consent. Thanks to our flexibility in research methods, we were able to respond to that demand and sharpened the focus of international legal research on the interface between prior assessments, consent and benefit sharing. Better understanding this interface proved a turning point in making a contribution to scholarship in international biodiversity and human rights law. ${ }^{39}$ In addition, this insight contributed to a political sociological research agenda on discursive spaces for local issues in the Convention on Biological Diversity, ${ }^{40}$ as well as reflections on the potential role of community protocols in local empowerment. ${ }^{41}$

Overall, the complexity of power relationships that may affect and shape research in different ways remains difficult to foresee at the research planning or bidding stage, even where due attention is paid to risk and contingency plans. ${ }^{42} \mathrm{An}$ explicit and iterative engagement with questions of power, in dialogue with research participants, enables the adjustment of research questions, knowledge exchange approaches, and research methods. Such adjustments in turn support research ethics and shape research findings and outreach in ways that might be useful to all research partners.

\subsection{Consent}

A final practical issue in dealing with power in our research stemmed from the gap that persists between formal institutional ethics procedures and the kinds of iterative ethical

\footnotetext{
${ }^{38}$ Fisher et al., n. 9 above; E. Tsioumani, 'Beyond Access and Benefit-sharing: Lessons from the Law and Governance of Agricultural Biodiversity’ (2018) 21 The Journal of World Intellectual Property, pp. 106-122.

T. ${ }^{39}$ E. Morgera, 'Under the Radar: Fair and Equitable Benefit-sharing and the Human Rights of Indigenous Peoples and Local Communities related to Natural Resources' (2019) International Journal of Human Rights DOI: 10.1080/13642987.2019.1592161. See also P. Marchegiani, E. Morgera \& L. Parks, 'Indigenous peoples' rights to natural resources in Argentina: the challenges of impact assessment, consent and fair and equitable benefit-sharing in cases of lithium mining' (SSRN 2019) BENELEX Working Paper No 19. Available at SSRN: https://ssrn.com/abstract=3317375.

${ }^{40}$ Convention on Biological Diversity, 5 June 1992, 1760 UNTS. 79.

${ }^{41}$ Parks, n. 22 above; Parks, n. 17 above; L. Parks and M. Schroder, 'What we talk about when we talk about 'local' participation in international biodiversity law. The changing scope of Indigenous peoples and local communities' participation under the Convention on Biological Diversity' (2018) 11

Participation and Conflict, pp. 743-785.

${ }^{42}$ Vermeylen \& Clark, n. 32 above.
} 
decisions that need to be taken in empirical research. This is clear from the example of funders' requirements on written consent, including requirements for benefit sharing from academic research. ${ }^{43}$ Little attention, however, had been paid to benefit sharing from non-commercial research in international law prior to our research. ${ }^{44}$

We did obtain written consent from all our research participants and strongly believe that meaningful consent should be a central part of a conversation where all involved in the research negotiate benefits, methods, and matters around consent including anonymity. ${ }^{45}$ But the suitability of a 'one size fits all' approach of information pamphlets and consent forms that have obtained prior approval from ethics committees deserves some discussion in light of the iterative ethics considerations we experienced in our research. These considerations include researchers' evolving understanding of the concrete needs and demands for sharing benefits arising from their research through an ongoing dialogue with all research partners. This led to the realization that such a dialogue should include ethics committees.

The pre-approved forms and information pamphlets, developed in line with funders' ethical guidance and specific advice, were perfectly suited to some participants, most often representatives of various authorities. But this was not always true in community settings as revealed in the application of our research methods. Where our method was participant observation, we supplemented this pre-approved approach with oral presentations of our project, followed by time for the community to discuss and decide whether to allow us to observe their meetings as a group. Where we held interviews with individuals, the consent forms and pamphlets often proved off-putting for participants and we again supplemented with dialogue. In some cases, we added new information pamphlets when research partners and other advisors found the language we used inaccessible - our comparative approach was the origin of this challenge, since this limited our opportunities to co-develop forms with local actors. ${ }^{46}$

\footnotetext{
${ }^{43}$ See European Commission, 'Ethics' (2019), available at http://ec.europa.eu/research/participants/docs/h2020-funding-guide/cross-cutting-issues/ethics_en.htm. ${ }^{44}$ E. Morgera 'Fair and Equitable Benefit-sharing at the Crossroads of the Human Right to Science and International Biodiversity Law' (2015) 4 Laws, pp. 803-831.

${ }^{45}$ Pittaway, Bartolomei \& Hugman, n. 20 above.

${ }^{46}$ Massey University, 'A Brief Introduction to Te Ara Tika' (undated), available at: https:/www.massey.ac.nz/massey/fms/Human\%20Ethics/Documents/Te\%20Ara\%20Tika\%20summar y.pdf?91A1B6C1CCBE36D7116F20C62124D4EB.
} 
Our experiences with consent forms and the need to place these within a more holistic approach to ethical research also overlapped with the findings of our project on global environmental law. International guidance stresses the iterative nature of seeking consent and its intertwining with benefit sharing. ${ }^{47}$ The second trip to each research site offered important opportunities in that sense, as it allowed us to share interim research results with interested communities and to seek guidance on preferred outputs and formats. ${ }^{48}$ This aligned with the preferred way to share benefits from our own research. We received some positive feedback in this regard. Nevertheless, it was difficult for us to remain in touch in an effective manner with communities in between and after trips, which was another limitation of the research. We mainly relied on partner NGOs as a channel both for ongoing communications with communities, and as a guarantor that communities could always get hold of us if needed, which gave more meaning to the right to withdraw from the project. It remains to be seen if this is a viable way to continue the research relationship beyond the life of the project.

Our main finding in attempting to apply the spirit of PAR in a research project involving many different research partners in five different cases is thus that ethical considerations at research-design stage are unlikely to cover all the questions that will arise during actual research. This cannot, in practice, be separated from the application of (and need to adapt) the chosen research methods. These inter-linkages draw into question pre-determined practices for seeking consent and sharing benefits from academic research, or pre-determined research directions. An iterative approach is instead needed to allow researchers to better understand and negotiate these matters in

\footnotetext{
${ }^{47}$ See, e.g., Mo'otz Kuxtal Voluntary Guidelines for the Development of Mechanisms, Legislation, or Other Appropriate Initiatives to Ensure the 'Prior Informed Consent', 'Free Prior Informed Consent' or 'Approval and Involvement', Depending on National Circumstances, of Indigenous People and Local Communities for Accessing Their Knowledge, Innovations and Practices, the Fair and Equitable Sharing of Benefits Arising from the Use and Application of Such Knowledge, Innovations and Practices for Reporting and Preventing Unauthorized Access to Such Knowledge, Innovations and Practices, CBD Decision XIII/18 [2016]. Note that our project was not intended to draw on the traditional knowledge of indigenous peoples and local communities, and we made an express commitment not to include references to any such knowledge inadertently revealed to us in our notes. We were interested in understanding the practical constraints and legal demands of these communities, and their understanding and experience of the role of international law, if any, in protecting their traditional knowledge and traditionally owned/used resources.

${ }^{48}$ Details of research outputs are available at: https://www.strath.ac.uk/research/strathclydecentreenvironmentallawgovernance/benelex/researchoutp uts/.
} 
a dialogue with all research partners. Ethics committees and research funders should thus also be involved in, or at least factor into their procedures the need for, these iterative dialogic practices.

\section{A SUMMARY OF EXPERIENCES AND REFLECTIONS}

The inter-linkages between research methods and ethics have not surfaced in the extant literature on global environmental law. Researchers, however, need to be alert to multiple and possibly interconnected power imbalances when legal concepts travel across different levels and sites, as well as to the possible impacts of their own research (and their own biases) on those imbalances. ${ }^{49}$ The central point arising from our experience is the need for iterative approaches to the choice of research methods and ethics, particularly in the context of local-level research. Not all questions can be answered in prior research designs and formal ethics procedures. An iterative approach can strengthen research design by providing opportunities to enrich and fine-tune research directions and research design to the needs of research participants.

Each research project is unique and will necessarily encounter different obstacles, issues, and opportunities. Our reflections, however, may be of use to other researchers who are considering comparative interdisciplinary approaches for the study of global environmental law. We would thus like to draw attention to what might be termed the living research process. ${ }^{50}$ Equally we would like to underscore the need to discuss trade-offs in designing research in global environmental law, particularly when it is comparative, externally funded, or subject to one-off research ethics advice.

In sum, as scholarship continues to evolve on transnational and global environmental law in particular, this short note recommends explicit reflection on the need for iterative research methods and a critical consideration of research ethics. Such reflection can

further enrich the already debated substantive questions about the nature and content of global environmental law and its methodologies.

\footnotetext{
${ }^{49}$ Morgera, n. 39 above.

${ }^{50}$ Reason \& Bradbury, n. 13 above.
} 
